\title{
Article \\ Sexual Experience Induces the Expression of Gastrin-Releasing Peptide and Oxytocin Receptors in the Spinal Ejaculation Generator in Rats
}

\author{
Takumi Oti ${ }^{1,2, *(D)}$, Ryota Ueda ${ }^{2}$, Ryoko Kumagai ${ }^{3}$, Junta Nagafuchi ${ }^{2}$, Takashi Ito ${ }^{2}$, Tatsuya Sakamoto ${ }^{2}$, \\ Yasuhiko Kondo ${ }^{3}$ and Hirotaka Sakamoto ${ }^{2, * \mathbb{D}}$
}

1 Department of Biological Sciences, Faculty of Science, Kanagawa University, Hiratsuka 259-1293, Kanagawa, Japan

2 Ushimado Marine Institute (UMI), Graduate School of Natural Science and Technology, Okayama University, Ushimado, Setouchi 701-4303, Okayama, Japan; pw1746ro@s.okayama-u.ac.jp (R.U.); pj5v7k6c@s.okayama-u.ac.jp (J.N.); ti1922@yamada-bee.com (T.I.); ryu@uml.okayama-u.ac.jp (T.S.)

3 Department of Animal Sciences, Teikyo University of Science, Uenohara 409-0193, Yamanashi, Japan; qup.kuma.po@gmail.com (R.K.); ykondo@ntu.ac.jp (Y.K.)

* Correspondence: sc419439@s.okayama-u.ac.jp (T.O.); hsakamo@okayama-u.ac.jp (H.S.); Tel.: +81-463-59-4111(ext. 2540) (T.O.); +81-869-34-5210 (H.S.)

check for updates

Citation: Oti, T.; Ueda, R.; Kumagai, R.; Nagafuchi, J.; Ito, T.; Sakamoto, T.; Kondo, Y.; Sakamoto, H. Sexual Experience Induces the Expression of Gastrin-Releasing Peptide and Oxytocin Receptors in the Spinal Ejaculation Generator in Rats. Int. J. Mol. Sci. 2021, 22, 10362. https:// doi.org/10.3390/ijms221910362

Academic Editor: Diego Guidolin

Received: 15 August 2021

Accepted: 23 September 2021

Published: 26 September 2021

Publisher's Note: MDPI stays neutral with regard to jurisdictional claims in published maps and institutional affiliations.

Copyright: (c) 2021 by the authors. Licensee MDPI, Basel, Switzerland. This article is an open access article distributed under the terms and conditions of the Creative Commons Attribution (CC BY) license (https:// creativecommons.org/licenses/by/ $4.0 /)$.

\begin{abstract}
Male sexual function in mammals is controlled by the brain neural circuits and the spinal cord centers located in the lamina X of the lumbar spinal cord (L3-L4). Recently, we reported that hypothalamic oxytocin neurons project to the lumbar spinal cord to activate the neurons located in the dorsal lamina $X$ of the lumbar spinal cord $(\mathrm{d} X \mathrm{~L})$ via oxytocin receptors, thereby facilitating male sexual activity. Sexual experiences can influence male sexual activity in rats. However, how this experience affects the brain-spinal cord neural circuits underlying male sexual activity remains unknown. Focusing on dXL neurons that are innervated by hypothalamic oxytocinergic neurons controlling male sexual function, we examined whether sexual experience affects such neural circuits. We found that $>50 \%$ of $d X L$ neurons were activated in the first ejaculation group and $\sim 30 \%$ in the control and intromission groups in sexually naïve males. In contrast, in sexually experienced males, $\sim 50 \%$ of $\mathrm{dXL}$ neurons were activated in both the intromission and ejaculation groups, compared to $\sim 30 \%$ in the control group. Furthermore, sexual experience induced expressions of gastrin-releasing peptide and oxytocin receptors in the lumbar spinal cord. This is the first demonstration of the effects of sexual experience on molecular expressions in the neural circuits controlling male sexual activity in the spinal cord.
\end{abstract}

Keywords: sexual experience; lumbosacral spinal cord; spinal ejaculation generator; brain-spinal cord neural circuits; gastrin-releasing peptide; oxytocin; male sexual activity

\section{Introduction}

Mammalian male sexual function is controlled not only by brain neural circuits but also by spinal cord centers, including the spinal ejaculation generator (SEG) located in the lamina X, and a portion of VII of the upper lumbar spinal cords (L3 and L4 levels) [1,2]. The primary component of SEG is a population of neurons called lumbar spinothalamic (LSt) neurons, which are believed to function to convey peripheral signals to the brain through direct projection from the lumbar spinal cord to the thalamus [1]. Specific toxin treatments that selectively destroy LSt neurons completely eliminate ejaculation in rats [1]. LSt neurons are much more prominent in male rats than in female rats and they are involved in the expression of galanin [1-3], cholecystokinin [4,5], enkephalin [6], and gastrin-releasing peptide (GRP) [7].

We reported in our previous study that neurons located in the dorsal lamina $X$ of the lumbar spinal cord (dXL) overlapped with SEG project axons to the parasympathetic 
nervous system in the lower lumbar and upper sacral spinal cords (at the L5-L6 and S1 levels), the sacral autonomic nucleus (SAN), which controls male sexual functions such as erection and ejaculation in mice [8], rats [7,9], Suncus [10], and macaque monkeys [11]. Hypothalamic neurons that express nonapeptide oxytocin (OXT) have been reported to send axons not only to the brain region but also to the lower spinal cord-the SAN and the somatic spinal nucleus of the bulbocavernosus (SNB) that innervate the bulbocavernosus muscle attached to the base of the penis. It has already been suggested that these OXT projections are involved in penile function [12,13]. It is therefore considered plausible that the $\mathrm{dXL}$ and SNB systems, which are localized at similar levels in the lumbosacral spinal cord, have direct and/or indirect interaction with each other to modulate male sexual functions. The dXL neurons appear to generate ejaculation behavior by synchronizing both autonomic and somatic centers such as SAN and SNB in the lumbosacral spinal cord. Recently, we had reported that hypothalamic OXT neurons project to the upper lumbar spinal cord and activate lumbar neurons that express OXT receptors (OXTRs) and then facilitate male sexual functions such as erection and ejaculation [14].

Substantial evidence supports the claim that the first sexual experience significantly facilitates male sexual activity in rats [15]. Sexual experience is essential in indicating a positive preference for odors in estrous females [16]. The lesion of the medial amygdala in sexually naïve male rats severely impairs copulatory behavior, but not in sexually experienced males $[17,18]$. Sexual experience is also known to affect synaptic plasticity and spinogenesis in the medial preoptic area (mPOA) [19,20]. Furthermore, it has been reported that sexual experience increases the Oxtr expression in the mPOA and ventromedial hypothalamus of male rats [21]. These results suggest that sexual experience promotes male sexual activity through altering gene expression such as OXT and OXTR of the neural circuits that control male sexual activity in the hypothalamus.

In this study, we focused on dXL neurons that are innervated by hypothalamic OXTergic neurons controlling male sexual function and examined whether sexual experience affects such male-specific neural circuits in the spinal cord. Here, we demonstrated that sexual experience in male rats alters the expression levels of GRP and OXTR in dXL neurons.

\section{Results}

\subsection{Experiment 1: Changes in the Activation of dXL Neurons between Naïve and Sexually Experienced Male Rats}

Focusing on sexual experience or the lack of it, some experiments were conducted, as indicated in Figure 1a, so as to analyze the activation of dXL neurons after sexual behavior in male rats. Male rats were immediately separated after their first mount with the penile insertion to the vagina (intromission) as an index of erection (the 1st-Intromission group), or the male rats were sacrificed immediately after the first ejaculation (the 1stEjaculation group). The male rats were perfusion fixed for $15 \mathrm{~min}$ after each behavior. The control rats were placed in the observation cage, and they were not presented with estrous females. Next, the rats that ejaculated two or more times in the three mating sessions were designated as experienced males (the sexually experienced group). The experienced male rats were separated immediately after 9 intromissions or the first ejaculation. The male rats were perfusion fixed for $15 \mathrm{~min}$ after each behavior. For the experienced male rats, a control group was also maintained, in which the estrous female rat was not presented in the observation cage as in the inexperienced group. Double immunofluorescence was performed for each spinal cord sample using antibodies against GRP, a marker for dXL neurons, and phosphorylated extracellular signal-related kinases 1 and 2 (pERK), which is a marker protein for neural activation (Figure 1b,c). The expression of pERK (magenta) in GRP-positive $\left(^{+}\right)$neurons (green) was observed only in the first ejaculated rats (the 1st-Ejaculation group), and almost no activation of $\mathrm{GRP}^{+}$neurons was recorded in the first intromission rats (the 1st-Intromission group) (Figure 1b,d; Table 1). In contrast, in the experienced male rats, $\mathrm{GRP}^{+}$neurons were activated in both the Intromission and Ejaculation groups, and the ratio of activated $\mathrm{GRP}^{+}$neurons (the number of $\mathrm{pERK}^{+}$cells in 
the $\mathrm{GRP}^{+}$cells) was significantly higher in both the Intromission and Ejaculation groups relative to those in the Control group (two-way analysis of variance (ANOVA), $F_{2,28}=4.10$, ${ }^{*} p<0.05$ vs. Control, $+p<0.05$ vs. Intromission. Figure 1c,e; Table 1). For the experienced male rats, no statistical difference was noted in the ratio of activated $\mathrm{GRP}^{+}$neurons in the Intromission and Ejaculation groups (Table 1).

a Experiment 1

15 min after intromission or ejaculation

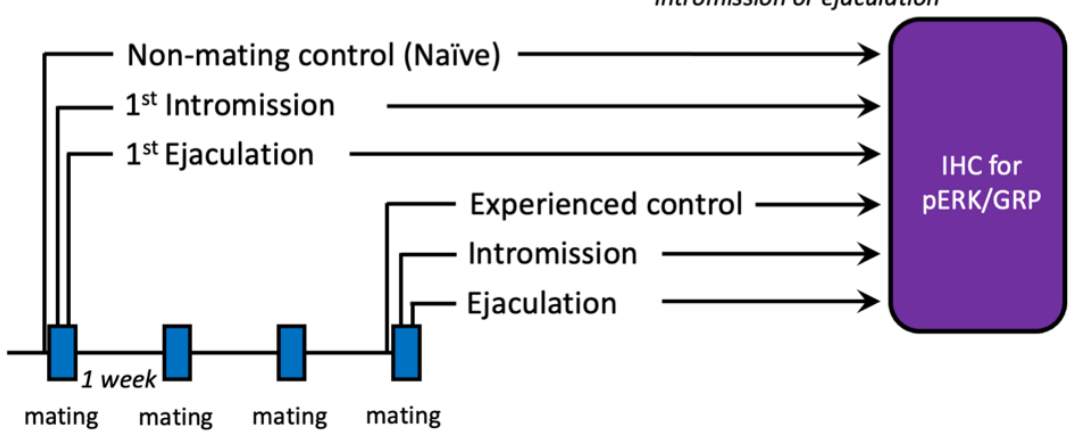

b

\section{Naïve}

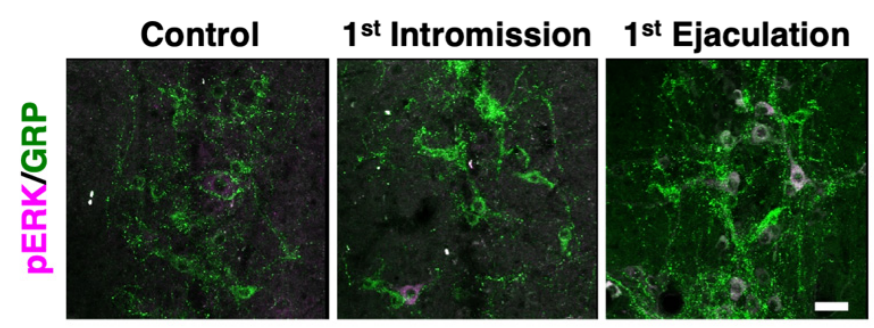

C

\section{Experienced}

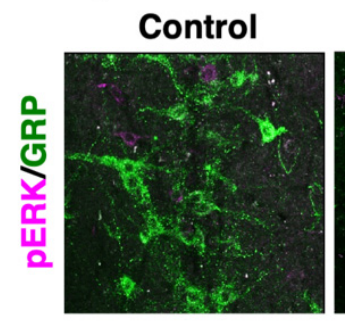

\section{Intromission}

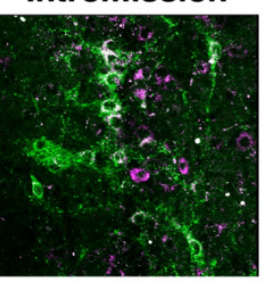

d

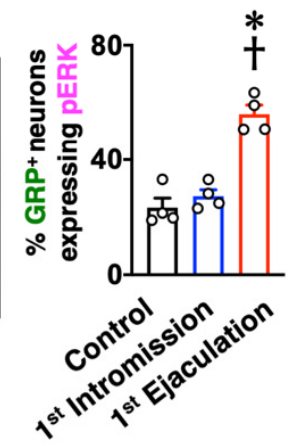

e
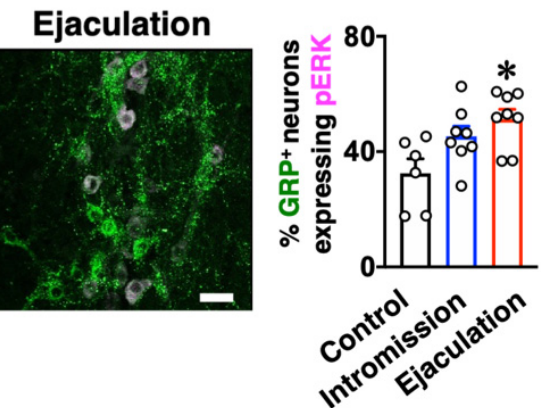

Figure 1. (a) Experimental paradigm of Experiment 1: changes in the activation of $d X L$ neurons between naïve and sexually experienced male rats. Activation of spinal GRP-positive $\left(^{+}\right)$neurons after sexual behavior in male rats with or without sexual experience. (b) Expression of phosphorylated extracellular signal-related kinases 1 and 2 (pERK) (magenta) in spinal GRP ${ }^{+}$neurons (green) after sexual behavior of sexually naïve males. Left panel, Control. Middle panel, 1st-Intromission group. Right panel, after 1st Ejaculation. (c) Expression of pERK (magenta) in spinal GRP ${ }^{+}$neurons (green) after sexual behavior of sexually experienced males. Left panel, Control. Middle panel, mounts and intromission only. Right panel, after ejaculation. (d) In naïve males, ejaculation significantly increased 
pERK expression in $\mathrm{GRP}^{+}$neurons compared to that in the Control and Intromission groups (data presented as mean \pm standard error of the mean (SEM) and individual dots; $n=4$ per group). (e) In experienced males, Ejaculation significantly increased pERK expression in $\mathrm{GRP}^{+}$neurons compared to that in the Control and Intromission groups (data presented as mean \pm SEM and individual dots; $n=6$ in Control group, $n=8$ in the Intromission group, $n=8$ in the Ejaculation group). No differences in any of the parameters were observed between the groups with or without sexual experience (two-way ANOVA, $F_{2,28}=4.10,{ }^{*} p<0.05$ vs. Control, $+p<0.05$ vs. Intromission). Scale bar: $50 \mu \mathrm{m}$.

Table 1. The proportion of pERK expression in GRP-immunoreactive neurons (\%) after sexual behavior.

\begin{tabular}{cccc}
\hline & Control & Intromission & Ejaculation \\
\hline Naïve & $23.3 \pm 3.3$ & $27.3 \pm 2.2$ & $56.1 \pm 3.2$ \\
Experienced & $32.5 \pm 5.0$ & $45.3 \pm 3.5$ & $51.3 \pm 3.4$ \\
\hline
\end{tabular}

\subsection{Experiment 2: Effects of Sexual Experience on the dXL Neuron System}

Male rats who ejaculated for the first time (the first ejaculation) during mating and gained sexual experience were fixed 1 week after their first ejaculation (1st-Ejaculation group) (Figure 2a). In the control group, naïve male rats were placed in the observation cage but they were not presented with any estrous females. We examined changes in several molecular expressions (i.e., GRP, OXTR, and OXT) activated by the first ejaculation (sexual experience) in dXL neurons. Oxtr-yellow fluorescent protein (YFP) transgenic (Tg) rats [14] were used to histologically analyze the expression of OXTR. GRP-immunoreactivity (green) was higher in the 1st-Ejaculation group than in the Naïve group (unpaired $t$-test, $t_{6}=2.65$, $* p<0.05$ vs. Naïve, Figure $2 b, c$ ). The expression of Oxtr (red) was also higher in the 1st-Ejaculation group than in the Naïve group (unpaired $t$-test, $t_{6}=3.42$, ${ }^{*} p<0.05 \mathrm{vs}$. Naïve, Figure $2 \mathrm{~b}, \mathrm{~d})$. In contrast, no obvious differences were noted in OXT-immunoreactivity (Cyan) (unpaired $t$-test, $t_{6}=0.32, p=0.76$, Figure $2 \mathrm{~b}$,e). In addition, no obvious effects of sexual experience were noted on the expression of Oxtr in GRP ${ }^{+}$neurons (unpaired $t$-test, $t_{6}=1.51, p=0.18$, Figure $\left.2 b, f\right)$. No effects of sexual experience were observed on either the number of $\mathrm{GRP}^{+}$neurons or the $\mathrm{Oxtr}^{+}$neurons between the Naïve group and the 1st-Ejaculation group (unpaired $t$-test, $t_{6}=1.51, p=0.18$ in $\mathrm{f}, t_{6}=1.90, p=0.11$ in $\mathrm{g}$, Figure 2g,h). Next, changes in Grp mRNA and Oxtr mRNA expression after sexual experience were analyzed through the real-time quantitative PCR (qPCR) technique, where the Naïve group was placed in the observation cage alone, and the 1st-Ejaculation group was separated immediately after the first ejaculation. The rats were sacrificed $60 \mathrm{~min}$ after ejaculation (Figure 2a). As a result, no obvious changes in the mRNA expressions of Grp and Oxtr were observed in the lumbar spinal cord (L3-L4 level; somal region of dXL neurons) (Figure 3). Furthermore, we analyzed GRP ${ }^{+}$projections to the SAN involved in the parasympathetic function in the spinal cord. GRP-immunoreactivity in the SAN was found to be significantly higher in the 1 st-Ejaculation group (unpaired $t$-test, $t_{7}=2.30$, $p<0.05$, Figure 4). 
a

Experiment 2

1 week after $1^{\text {st }}$ ejaculation

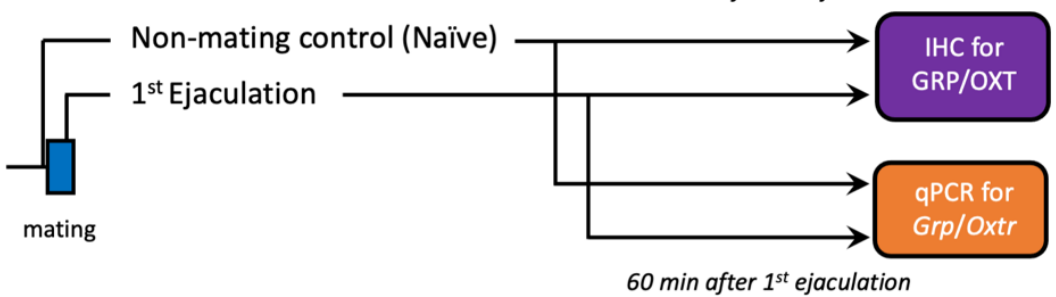

b
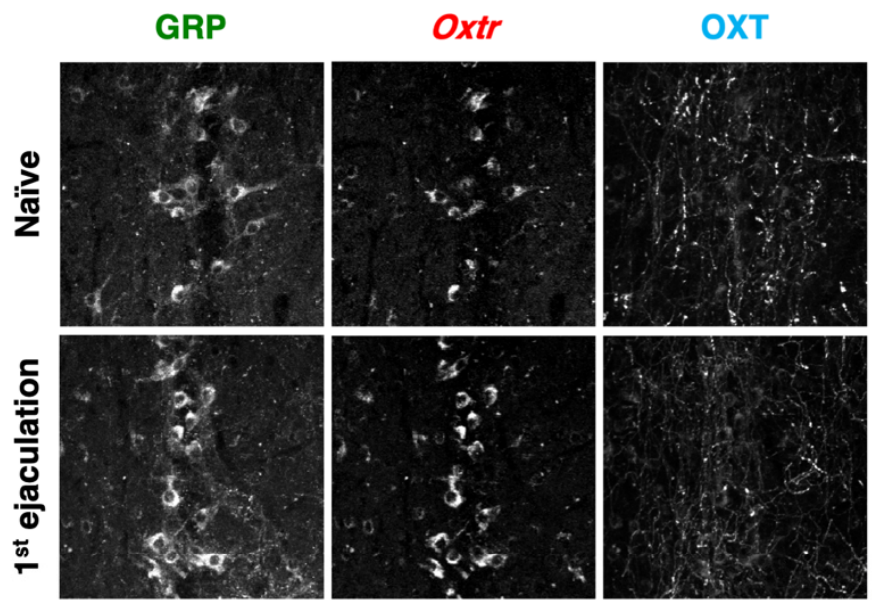

\section{GRP/OxtHOXT}
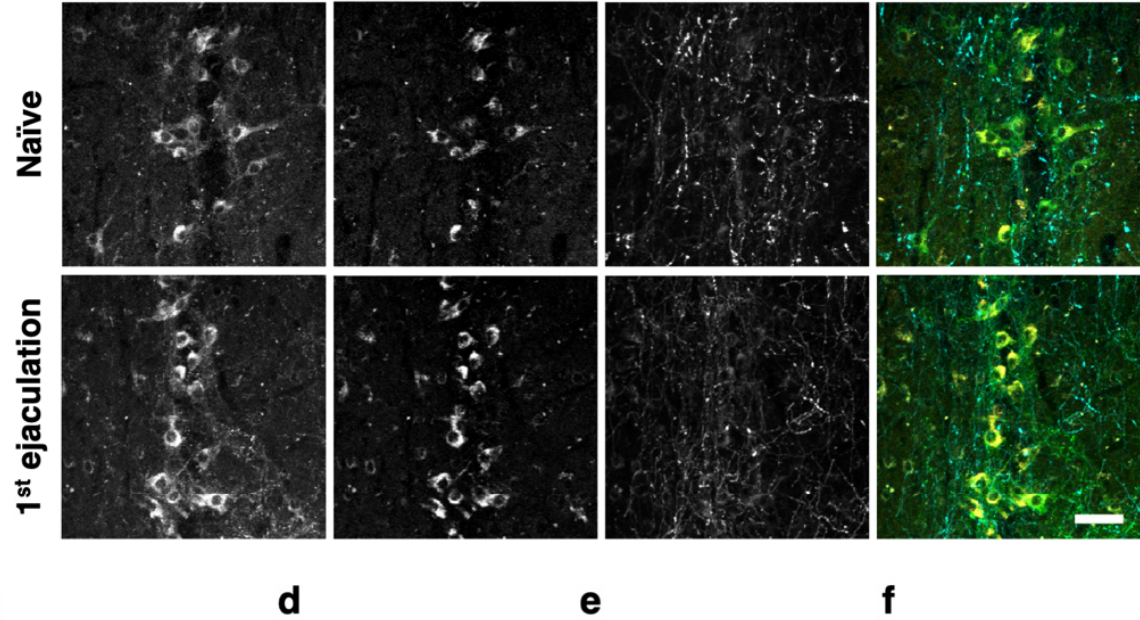

e

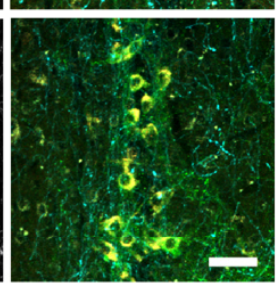

C

C

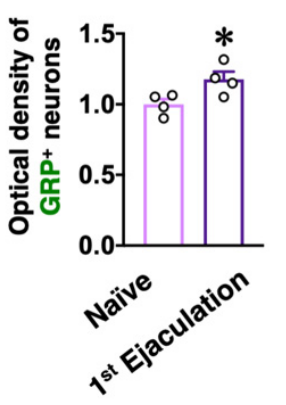

g

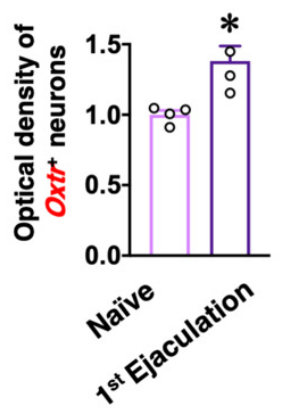

h
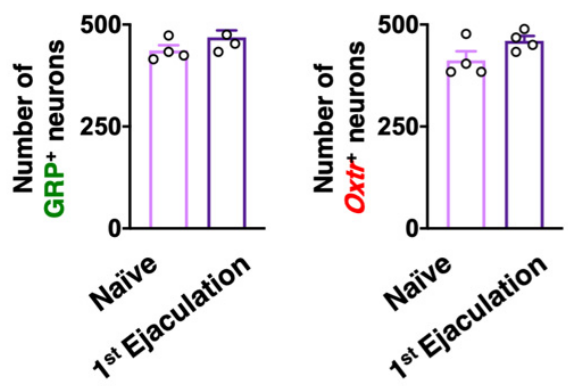

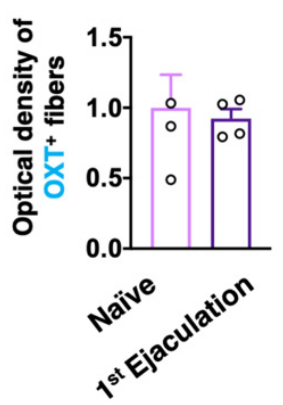

f

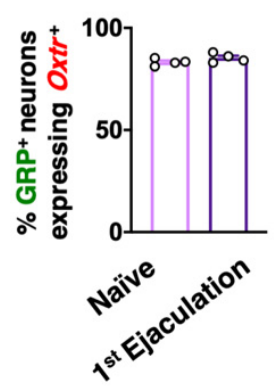

Figure 2. (a) Experimental paradigm of Experiment 2: effects of sexual experience on the dXL neuron system. (b) Immunohistochemistry for GRP and OXT in the upper lumbar spinal cord of Oxtr-YFP transgenic (Tg) male rats with or without sexual experience ( $n=4$, respectively). GRPimmunoreactivity (green) and YFP signals $(\mathrm{Oxtr})$ (red) were higher in 1st-Ejaculation Tg male rats than in Naïve Tg male rats. No difference was noted in the intensity of OXT-positive $\left(^{+}\right)$fibers (Cyan). In this study, we detected the immunofluorescence of OXT-neurophysin as a marker for OXT neurons. 
Scale bar: $100 \mu \mathrm{m}$. (c) The intensity of $\mathrm{GRP}^{+}$neurons significantly increased in 1st-Ejaculation Tg male rats compared to Naïve $\mathrm{Tg}$ male rats (unpaired $t$-test, $t_{6}=2.65,{ }^{*} p<0.05$ vs. Naïve). (d) The intensity of $\mathrm{Oxtr}^{+}$neurons significantly increased in 1st-Ejaculation $\mathrm{Tg}$ male rats compared to Naïve $\mathrm{Tg}$ male rats (unpaired $t$-test, $t_{6}=3.42,{ }^{*} p<0.05$ vs. Naïve). (e) No statistical difference was noted in the intensity of $\mathrm{OXT}^{+}$fibers between 1st-Ejaculation and Naïve Tg male rats (unpaired $t$-test, $t_{6}=0.32$ ). $(\mathbf{f}-\mathbf{h})$ No statistical difference was noted in the ratio of $\mathrm{GRP}^{+} / \mathrm{Oxtr}^{+}(\mathbf{f})$, number of $\mathrm{GRP}^{+}$neurons $(\mathrm{g})$, or number of $\mathrm{Oxtr}^{+}$neurons (h) between 1st-Ejaculation and Naïve Tg male rats (unpaired $t$-test, $t_{6}=1.51$ in $\mathrm{f}, t_{6}=1.51$ in $g, t_{6}=1.90$ in $\mathrm{h}$ ).

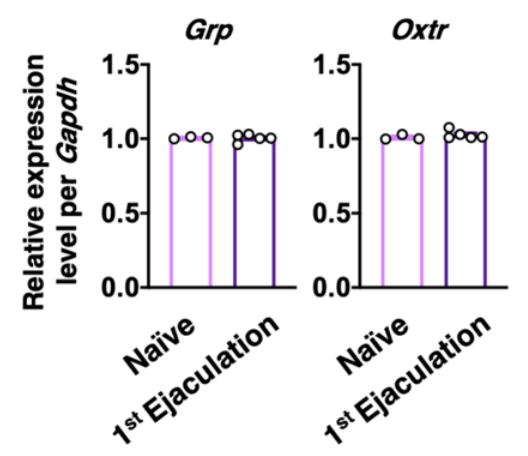

Figure 3. Effect of sexual experience on the Grp and Oxtr mRNA expression in the somal region of $\mathrm{dXL}$ neurons. No statistical difference was noted in the Grp and Oxtr mRNA expression between Naïve male rats $(n=3)$ and 1st-Ejaculation male rats $(n=5)$ (unpaired $t$-test, $t_{6}=0.15$ in Grp, $t_{6}=0.93$ in $O x t r$ ).
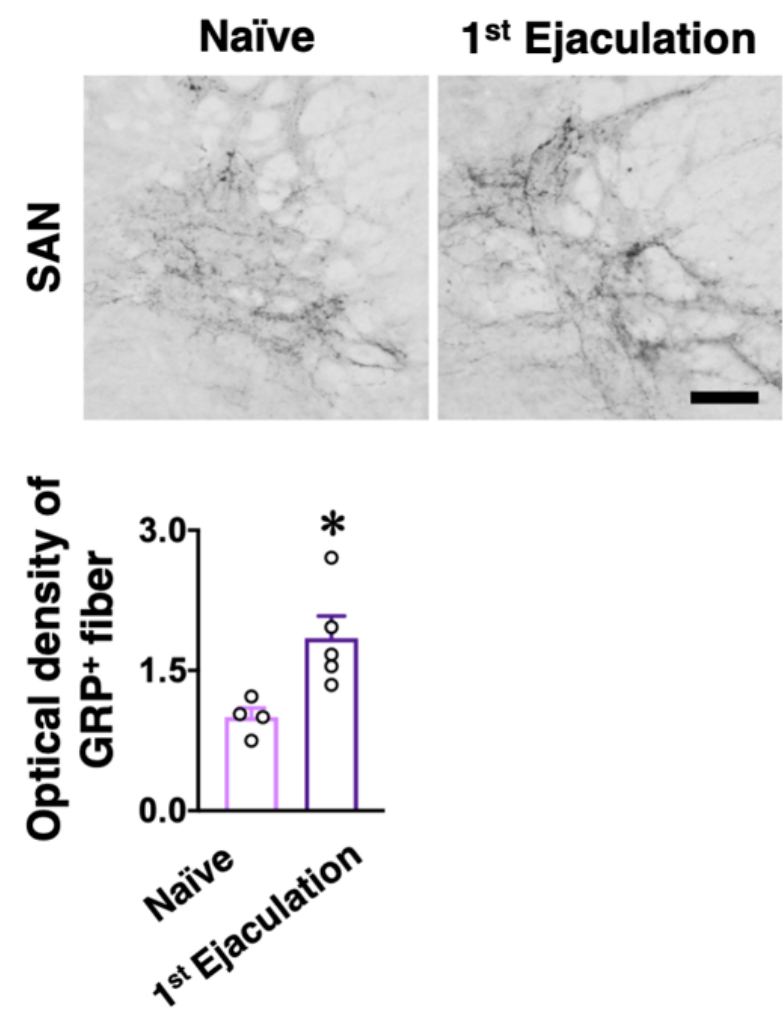

Figure 4. The effects of sexual experience on GRP-positive $\left(^{+}\right)$fibers in the SAN. (Upper panel) The $\mathrm{GRP}^{+}$fibers were higher in 1st-Ejaculation male rats $(n=5)$ than in Naïve male rats $(n=4)$. Scale bar: $50 \mu \mathrm{m}$. (Lower panel) Semi-quantitative analysis revealed that $\mathrm{GRP}^{+}$fibers were significantly increased in 1st-Ejaculation male rats than in Naïve male rats (unpaired $t$-test, $t_{7}=3.00,{ }^{*} p<0.05$ vs. Naïve). 


\subsection{Experiment 3: Evaluation of Whether Reflexive Erection or Ejaculation Activates dXL Neurons}

Finally, double immunofluorescence to determine the expressions of GRP and pERK was performed to evaluate the relationship between erection or ejaculation and the activation of dXL neurons. Male rats with three or more sexual behavior tests (with at least two ejaculations) were used in this study (Figure 5a). In the reflexive erection test, we investigated whether erections alone activated $\mathrm{dXL}$ neurons using male rats who were separated immediately after the third erection clusters. Male rats were perfusion fixed for $15 \mathrm{~min}$ after the last erection (Figure 5a). The control rats were gently restrained in the supine position with surgical tape, but their penises were not stimulated. Double immunofluorescence for GRP and pERK expression showed no significant effect of erections on the number of $\mathrm{GRP}^{+}$neurons expressing pERK (unpaired $t$-test, $t_{6}=0.05, p=0.96$, Figure $5 \mathrm{~b}$ and Table 2). We further evaluated the effect of ejaculation on the activation of $d X L$ neurons by using a $p$-chloroamphetamine (PCA)-induced ejaculation model [22] with almost no erection (Figure 5a). Ejaculation was inducible through intraperitoneal administration of PCA [22]. Most of the anesthetized male rats showed an ejaculatory reflex of approximately $6 \mathrm{~min}$ after PCA administration [22]. No significant effect of ejaculation on the number of $\mathrm{GRP}^{+}$ neurons expressing pERK was also detected (unpaired $t$-test, $t_{7}=1.60, p=0.15$, Figure $5 c$ and Table 2).

\section{a}

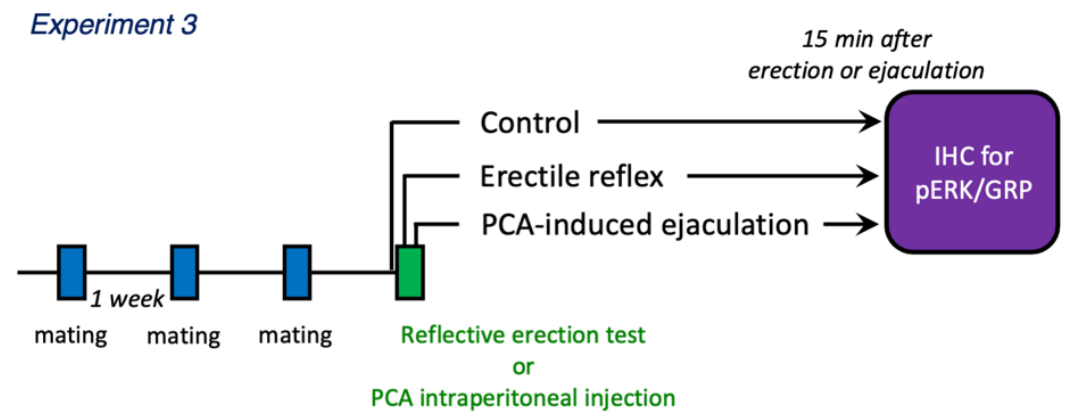

b

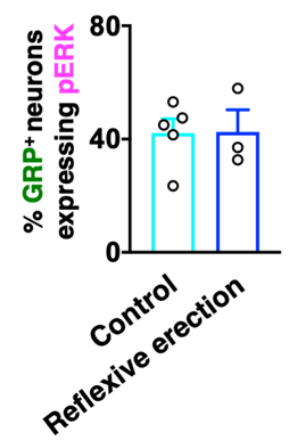

C

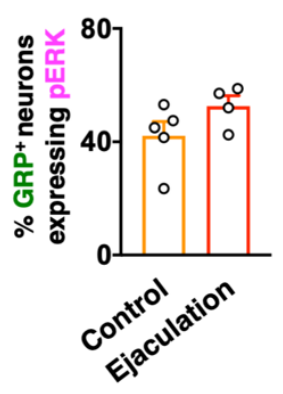

Figure 5. (a) Experimental paradigm of Experiment 3: evaluation of whether reflexive erection- or non-erection-induced ejaculation activates $\mathrm{dXL}$ neurons. (b) No statistical difference was noted in the ratio of activated spinal GRP-immunoreactive neurons between the Control $(n=5)$ and Reflexive erection groups $(n=3)$ (unpaired $t$-test, $\left.t_{6}=0.05\right)$. (c) There was no statistical difference in the ratio of activated spinal GRP-immunoreactive neurons between the Control $(n=5)$ and Ejaculation groups $(n=4)$ (unpaired $t$-test, $\left.t_{7}=1.60\right)$. 
Table 2. The proportion of pERK expression in GRP-immunoreactive neurons (\%) after reflexive erection or PCA-induced ejaculation.

\begin{tabular}{cccc}
\hline & Control & Erection & Ejaculation \\
\hline Reflexive erection & $37.8 \pm 5.6$ & $42.5 \pm 7.8$ & - \\
\hline PCA-induced ejaculation & $42.1 \pm 5.0$ & - & $52.6 \pm 3.7$ \\
\hline
\end{tabular}

\section{Discussion}

As already reported, sexual experience facilitates male sexual activity in rats [15]. To elucidate the mode of action in the spinal cord in addition to that in the brain, we examined the effects of sexual experience on the dXL neuron system controlling penile function at the spinal cord level (Figure 6). We found that approximately two-fold more GRP neurons were activated in the ejaculation group than in the control and intromission groups in naïve males. In contrast, in male rats with sexual experience, activation of GRP neurons increased by $60 \%$ in the ejaculation group and by $40 \%$ in the intromission group as compared with that in the control group. An increase in $\mathrm{Fos}^{+}$cell proportion after exposure to bedding soiled by an estrous female was recorded in the POA, the bed nucleus of the stria terminalis, and the nucleus accumbens in sexually experienced, but not in naïve, male rats [16]. Similarly, a different proportion of dXL neurons was activated depending on the level of sexual experience, suggesting that the spinal circuit for male sexual function is modulated by male sexual experiences such as intromission and ejaculation. $\mathrm{dXL}$ neurons have been known to control ejaculation via innervation to the SAN and spinal motor nuclei, which are involved in penile function [23]. They are also known to be regulated by efferent inputs from hypothalamic OXT [14]. Sexual experience may raise excitability in dXL neurons by these OXT-ergic efferents originating from the hypothalamus, consequently promoting male sexual activity through increased efficiency of innervation to the SAN and/or SNB. Alternatively, sexual experience may immediately switch the autonomic function from erection (parasympathetic) to ejaculation (sympathetic) by activating $\mathrm{dXL}$ neurons.

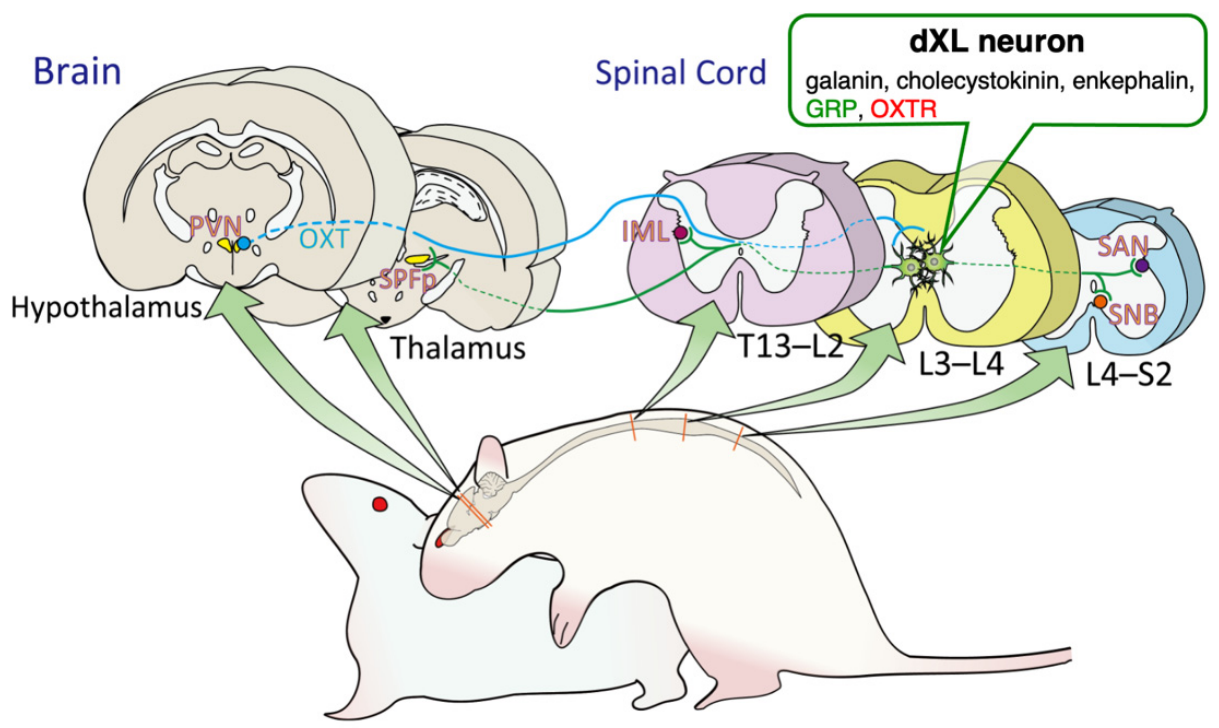

Figure 6. Schematic illustrations of the brain-spinal neural circuits that control male sexual activity in rats. Neurons located in the dorsal lamina $X$ of the lumbar spinal cord (dXL) express galanin, cholecystokinin, enkephalin, gastrin-releasing peptide (GRP), and oxytocin receptor (OXTR). dXL neurons can also be identified as lumbar spinothalamic (LSt) neurons projecting from the lumbar spinal cord to the thalamus. In addition, $\mathrm{dXL}$ neurons project to both parasympathetic (sacral autonomic nucleus [SAN]) and sympathetic (intermediolateral cell column [IML]) systems in the thoracic spinal cord (the T13-L2 level) preganglionic neurons and sexual motor neurons (the spinal nucleus of the bulbocavernosus, SNB) located in the lower lumbar and upper sacral spinal cord (the L4-S2 level). In the thalamus, the parvocellular portion of the subparafascicular thalamic nucleus (SPFp) may receive sexual information from the afferent inputs of spinal dXL neurons. 
In the mPOA of male rats, sexual experience induces the expression of 20 or more genes but reduces the expression of more than 10 genes [24]. Furthermore, sexual experience increases the expression of Oxtr mRNA in the mPOA and the ventromedial hypothalamus in male rats [21]. In dXL neurons, it is possible that sexual experience can increase OXTR expression at the protein level, although changes in the Oxtr mRNA level could not be observed. Similarly, GRP expression levels were increased by sexual experience in dXL neurons. Sexual experience also appeared to affect spinal neural circuits involved in male sexual activity. In sexually experienced rats, $\mathrm{d} X \mathrm{~L}$ neurons were activated not only by ejaculation but also by intromissions. On the other hand, in naïve rats, $\mathrm{dXL}$ neurons were activated only by ejaculation. The activation of $\mathrm{dXL}$ neurons during the first ejaculation (or the first sexual experience) may imply the occurrence of changes in gene expression patterns. In addition to GRP, galanin, cholecystokinin, and enkephalin are expressed in dXL neurons [1,3-7]. Sexual experience may alter the expressions of these neuropeptides. Transcriptome analysis of $\mathrm{dXL}$ neurons could reveal the comprehensive neural mechanisms of the effect of the first sexual experience on the SEG system and the modification of male sexual activity.

Spinal cord injury (SCI) at the thoracic level does not influence the level of galanin expression in dXL neurons, but it reduces GRP expression [25]. Different regulatory mechanisms may hence be involved in the expression of galanin and GRP in dXL neurons [25,26]. $\mathrm{SCI}$ in the upper spinal cord (e.g., the thoracic spinal cord) possibly eliminates efferent innervation to $\mathrm{dXL}$ neurons from the brain. The expression of GRP in $\mathrm{dXL}$ neurons appears to be affected by brain function because SCI at the thoracic level significantly reduced GRP expression in $\mathrm{dXL}$ neurons [25]. It is already well known that the spinal transection at the lower thoracic level significantly increases the number of reflexive penile erections because the nerve connection between the brain and the lumbosacral spinal cord is damaged and the erectile-suppressing effect from the brain does not function [27]. Therefore, hyperactivity in penile function may induce increased GRP expression in dXL neurons.

To separately evaluate the involvement of $\mathrm{dXL}$ neurons in erection and ejaculation, we applied the reflexive erection test and PCA to induce ejaculation. The ratio was kept at approximately $50 \%$, consistent with the ratio of activated $\mathrm{GRP}^{+}$neurons after intromissions or after ejaculation in sexually experienced rats (Figures 1 and 5; Tables 1 and 2). In a subpopulation of dXL neurons, male sexual experience may lower the burst threshold and increase its sensitivity to activation at ejaculation. $\mathrm{dXL}$ neurons project axons not only to the parasympathetic nervous system of the lumbosacral spinal cord (erection), but also to the sympathetic nervous system of the thoracic spinal cord (ejaculation) $[1,28]$. dXL neurons may be functionally orchestrating/switching between the parasympathetic and sympathetic systems, and, when the excitement level reaches a threshold, they become increasingly activated by erections to generate ejaculation. Further attention needs to be paid to behavioral studies using galanin-Cre and/or GRP-Cre mice combined with the AAV-gene delivery system (e.g., optogenetics, pharmacogenetics, and/or fiber photometry). These results together demonstrate the activation of $d X L$ neurons with a higher time resolution during a functional shift from erection (parasympathetic) to ejaculation (sympathetic).

\section{Materials and Methods}

All experiments were performed following the "Guiding Principles for Care and Use of Animals in the Field of Physiological Sciences" of the Physiological Society of Japan and were approved by the local Animal Experiment Committees of the Okayama University and the Teikyo University of Science. All efforts were made to minimize animal suffering and the number of animals used for the studies.

\subsection{Animals}

In experiment 1, adult male wild-type (Wt) rats of the Long-Evans strain (Institute for Animal Reproduction, Ibaraki, Japan) were used (Naïve: $n=4$ per group and Experienced: 
$n=6$ in Control group and $n=8$ in each Intromission and Ejaculation groups). In experiment 2, adult male Oxtr promoter-human heparin-binding epidermal growth factor-like growth factor (human diphtheria toxin receptor; Dxtr)-ChR2-YFP BAC (Oxtr-YFP) Tg rats [14] (Wistar strain) bred in the animal facilities of Okayama University were used for IHC analysis of the upper lumbar spinal cord ( $n=4$ each in Naïve and 1st-Ejaculation groups). Adult male Wt rats of the Wistar strain (Charles River Japan, Yokohama, Japan) were used for qPCR analysis ( $n=4$ in Naïve group and $n=5$ in 1st-Ejaculation group) and for IHC analysis of the lumbosacral spinal cord ( $n=3$ in Naïve group and $n=5$ in 1st-Ejaculation group). All rats were maintained on a $12 \mathrm{~h}$ light/dark cycle and provided with unlimited access to water and rodent chow.

\subsection{Effects of Sexual Experience on the Activation of dXL Neurons}

To obtain male rats that ejaculated for the first time or male rats that demonstrated intromission for the first time, a few male rats were separated immediately after their first mount with the penile insertion to the vagina (intromission) as an index of erection (the 1st-Intromission group), or they were sacrificed immediately after their first ejaculation (the 1st-Ejaculation group). The male rats were perfusion fixed for $15 \mathrm{~min}$ after each behavior (Figure 1a). The controls were placed in the observation cage, but they were not presented with estrous females. Furthermore, the rats who ejaculated two or more times in three mating sessions were designated as experienced males (the sexually experienced group) (Figure 1a). Experienced male rats were separated immediately after nine intromissions or their first ejaculations. Male rats were perfusion fixed for $15 \mathrm{~min}$ after each behavior. For the inexperienced male rats, a control group was prepared in which the estrous female rats were not presented in the observation cage as in the inexperienced group.

\subsection{Protein Expression of GRP, OXT, and YFP in the Lumbar Spinal Cord}

Male rats that ejaculated for the first time (the first ejaculation) during mating and thereby gained sexual experience were fixed 1 week after their first ejaculation (1stEjaculation group) (Figure 2a). In the control group, naïve male rats were placed in the observation cage and they were not presented with estrous female rats.

\subsection{Effects of Sexual Experience on the Grp and Oxtr mRNA Expressions in the Upper Lumbar Spinal Cord}

Changes in Grp mRNA and Oxtr mRNA expression after male sexual experience were analyzed by the quantitative PCR (qPCR) technique with the Naïve group placed in the observation cage alone, while the 1st-Ejaculation group was separated immediately after the first ejaculation. The rats were sacrificed $60 \mathrm{~min}$ after ejaculation under deep anesthesia with $100 \mathrm{mg} / \mathrm{kg}$ body weight of an intraperitoneal sodium pentobarbital injection for real-time qPCR analyses. RNA extraction and real-time qPCR analysis were performed according to our established methods [29]. Lumbar spinal cords (L3-L4 level) were quickly removed and placed on an ice bath. The dissected tissues were immediately frozen using powdered dry ice. The preparations were mounted in a cryostat (CM3050 S; Leica, Nussloch, Germany) and the lamina $X$ region was dissected along the rostrocaudal axis by using a stainless-steel needle (outer diameter, $1.2 \mathrm{~mm}$; inner diameter, $0.94 \mathrm{~mm}$ ). The dissected tissues were stored at $-80{ }^{\circ} \mathrm{C}$ until RNA extraction. Total RNA was extracted using the Illustra RNAspin Mini RNA Isolation Kit (GE Health Care, Buckingham, UK) according to the manufacturer's protocol. The concentration of total RNA was measured using the Qubit RNA Assay Kit (Thermo Fisher Scientific, Waltham, MA, USA). First-strand cDNA was synthesized from 250 ng of total RNA with random primers using the Omniscript RT Kit (Qiagen, Hilden, Germany). To assess Grp, Oxtr, and Gapdh expressions in the lumbar spinal cord of the Wt Wistar rats with or without sexual experience (Naïve; $n=3$, Experienced; $n=5$ ), a $15 \mu \mathrm{L}$ reaction volume consisting of 1X TaqMan Universal PCR Master Mix (Applied Biosystems, Foster, CA, USA), $300 \mathrm{nM}$ each of forward and reverse primers, and $200 \mathrm{nM}$ of TaqMan probe was used. We used the TaqMan qPCR methodology for rat Grp, Oxtr, and Gapdh (gene expression assays no. Rn00592059, Rn005635037_m1, and Rn99999916; amplicon 
lengths 75, 60, and 87, respectively). Amplification was performed at $95{ }^{\circ} \mathrm{C}$ for $10 \mathrm{~min}$ and 40 cycles with $95^{\circ} \mathrm{C}$ for $15 \mathrm{~s}$ and $60^{\circ} \mathrm{C}$ for $1 \mathrm{~min}$. The data of qPCR were analyzed using the CFX96 qPCR Detection System (Bio-Rad Laboratories, Hercules, CA, USA). The expression in each reaction was normalized by the expression of Gapdh as an internal control. Duplicate qPCR analysis was performed for each sample.

\subsection{The Activation of dXL Neurons after Reflexive Erection or PCA-Induced Ejaculation}

Male rats with three or more sexual training tests (with at least two ejaculations) were used in this study. In the reflexive erection test, we investigated whether erections alone could activate $\mathrm{dXL}$ neurons using male rats separated immediately after their third erection clusters. Male rats were perfusion fixed for $15 \mathrm{~min}$ after the last erection (Figure 5a). Control rats were gently restrained in the supine position by using surgical tape, but their penises were not stimulated. In addition, with reference to a previous study [22], PCA (C9635; Sigma, St. Louis, MO, USA; $20 \mathrm{mg} / \mathrm{kg}$ ) was intraperitoneally administered to sexually experienced rats in order to induce ejaculation with almost no erection. The rats, 15 min after ejaculation, were quickly perfusion fixed for immunohistochemical analyses.

\subsection{Sexual Behavior Test}

For sexual behavior tests, stimulus female rats were ovariectomized, and estradiol benzoate (5 $\mathrm{gg} / 0.1 \mathrm{~mL}$ sesame oil) was subcutaneously injected 2 days prior to testing. Progesterone (500 $\mu \mathrm{g} / 0.1 \mathrm{~mL}$ sesame oil) was subcutaneously injected $4-6 \mathrm{~h}$ before testing so as to induce sexual receptivity. Sexual behavior tests were performed for $30 \mathrm{~min}$, and the latency of the first mount, intromission, and ejaculation, as well as the number of mounts, intromissions, and ejaculations, were enumerated.

\subsection{Immunohistochemistry and Immunofluorescence}

The experimental rats were deeply anesthetized with intraperitoneal injections of sodium pentobarbital (50 $\mathrm{mg} \mathrm{kg}^{-\mathrm{h}}$ body weight) and perfused via the left ventricle with $100 \mathrm{~mL}$ of physiological saline, followed by $200 \mathrm{~mL}$ of $4 \%$ formaldehyde in $0.1 \mathrm{M}$ phosphate buffer (PB; pH 7.4). The spinal cords were immediately removed and post-fixed in the same fixative agent for $3 \mathrm{~h}$ at room temperature. After immersion in $25 \%$ sucrose in $0.1 \mathrm{M} \mathrm{PB}$ for $48 \mathrm{~h}$ at $4{ }^{\circ} \mathrm{C}$ for cryoprotection, the lumbosacral spinal cords were quickly frozen using powdered dry ice and then cut into $30 \mu \mathrm{m}$-thick horizontal (the lumbar spinal cord: L3-L4 level) and cross- (brain and the lumbosacral spinal cord: L5-S1 level) sections on a cryostat. The endogenous peroxidase activity of the samples was eliminated by incubation in $1 \% \mathrm{H}_{2} \mathrm{O}_{2}$ in absolute methanol for $30 \mathrm{~min}$, followed by three 5 min rinsing sessions with phosphate-buffered saline (PBS, pH 7.4). This $\mathrm{H}_{2} \mathrm{O}_{2}$ treatment was omitted for immunofluorescence. After blocking nonspecific binding with $1 \%$ normal goat serum and 1\% BSA in PBS containing 0.3\% Triton X-100 for $30 \mathrm{~min}$ at room temperature, the sections were then incubated with the primary rabbit antiserum against GRP (1:2000 dilution) (11081; AssayPro, St. Charles, MO, USA, Research Resource Identifier (RRID): AB_2571636) as described elsewhere [14,29-31]. In this study, we performed immunostaining for GRP as the marker for $\mathrm{dXL}$ neurons. The specificity of the anti-GRP serum in the spinal cord was performed as demonstrated elsewhere [31]. Immunoreactive products were detected with the Streptavidin-biotin Kit (Nichirei, Tokyo, Japan), followed by diaminobenzidine (Dojindo, Kumamoto, Japan) development according to our method detailed previously [7,11,14,30-32]. To determine the density of $\mathrm{GRP}^{+}$fibers in the lumbosacral spinal cord (L5-S1 level), at least ten sections per animal were analyzed using ImageJ software (ImageJ 1.44p, RRID_SCR_003070) with a set threshold level. The GRP ${ }^{+}$fiber pixel density was quantified as the average pixel density in the three regions-the SAN and dorsal horn (DH) of each animal, which was calculated as the ratio to the density in the DH in controls in each analysis. The results obtained indicate that the expression of GRP in the spinal DH was involved in the transmission of the itch sensation [31,33]; this distribution of GRP showed no difference with the sex of the subject $[7,14]$. 
To examine the activation of $\mathrm{GRP}^{+}$neurons, double immunofluorescence staining for GRP and phosphorylated extracellular signal-related kinases 1 and 2 (pERK), a marker protein for neuronal activation, was performed. After blocking nonspecific binding, as described earlier, the sections were incubated with the anti-pERK (1:1000 dilution) (\#9101; rabbit polyclonal antibody, Cell Signaling Technology, Danvers, MA, USA, RRID: AB_331646) $[14,34]$ overnight at $4{ }^{\circ} \mathrm{C}$. After rinsing with PBS, the sections were incubated for $1 \mathrm{~h}$ at room temperature with the Dylight 549-linked Fab Fragment Goat Anti-rabbit IgG (1:100 dilution) (Jackson Laboratory, Bar Harbor, ME, USA) [14]. After rinsing, the sections were incubated with $1 \% \mathrm{H}_{2} \mathrm{O}_{2}$ in absolute methanol for 20 min at room temperature. After blocking nonspecific binding, the sections were immersed overnight at $4{ }^{\circ} \mathrm{C}$ in a 1:200 dilution of GRP antiserum. The second-primary immunoreaction was visualized as green after a $1 \mathrm{~h}$ incubation with Alexa Fluor 488-linked anti-rabbit IgG (1:2000 dilution) (Molecular Probes, Eugene, OR, USA, RRID: AB_2576217) [7,9,14].

To determine the distribution of OXT-containing axons, triple immunofluorescence staining was performed for GRP (1:1000 dilution) and OXT-neurophysin (PS60; mouse monoclonal antibody, RRID: CVCL_G254) (1:1000 dilution)—a marker protein for OXT neurons. The PS60 antibody has previously been shown to be specific for OXT neurons at the ultrastructure level [35-37]. The Alexa Fluor 555-linked anti-rabbit IgG raised in goats (Molecular Probes, RRID: AB_2535850) [29] and Alexa Fluor 633-linked antimouse IgG raised in goats (Molecular Probes, RRID: AB_2535719) were used for detection at a 1:1000 dilution.

\subsection{Morphological Analysis}

We determined the proportion of $\mathrm{pERK}^{+}, \mathrm{GRP}^{+}$, and $\mathrm{YFP}^{+}$neurons in the lumbosacral spinal cord. Immunofluorescence analysis of pERK, GRP, and YFP expression in the neurons of the anterior lumbar spinal cord (L3-L4 level) was performed as described earlier using horizontal sections (approximately 18-22 $30 \mu \mathrm{m}$-thick sections per animal) [29,30,32]. Briefly, we counted the number of $\mathrm{pERK}^{+}, \mathrm{GRP}^{+}$, and $\mathrm{YFP}^{+}$cell bodies at $\times 200$ magnification in all sections and analyzed a $600 \mu \mathrm{m}^{2}$ area localized to the midline at the center. Subsequently, we acquired 5-15 micrographs per section, depending on the distribution of the $\mathrm{GRP}^{+}$neurons. These digital micrographs were selected and processed using Adobe Photoshop (Adobe Systems, San Jose, CA, USA) and printed at 300 dots per inch on photographic paper. GRP and YFP neurons were identified by their following characteristics: densely immunostained, anatomical localization (mainly dorsal, dorsolateral, or both to the central canal in the lamina $X$ of the lumbar segments III-IV), relatively large cell bodies (diameters approximately 20-30 $\mu \mathrm{m}$ ), and clear round nuclei (diameters approximately 10-15 $\mu \mathrm{m}$ ). To avoid overestimating the cell numbers, only $\mathrm{pERK}^{+}$or $\mathrm{YFP}^{+}$and $\mathrm{GRP}^{+}$ neurons that contained a round, transected nucleus were counted. As the mean diameter of the nuclei in the GRP neurons was much smaller than the $30 \mu \mathrm{m}$-thick sections, this analysis reduced the overestimation of the number of neurons. All micrographs were coded and evaluated without the knowledge of the experimental group designation, and the code was not broken until the analysis was complete.

We next performed a semi-quantitative analysis of the GRP, YFP, and OXT expression. To determine the density of $\mathrm{GRP}^{+}$and $\mathrm{YFP}^{+}$somata and $\mathrm{OXT}^{+}$fibers in the lumbar spinal cord (L3-L4 level), at least 15 horizontal sections (30 $\mu \mathrm{m}$ thick) per animal were captured (magnification, $\times 42$ per section). The optical density of the YFP signals was determined using black-and-white images that were converted from micrographs using ImageJ software (Image J 1.44p; National Institutes of Health, Bethesda, MD, USA) according to our established methods [23,30,32]. Briefly, the optical density of the intrinsic fluorescence was estimated by comparison with similar areas of the control sections. The $\mathrm{GRP}^{+}$and $\mathrm{YFP}^{+}$ soma pixel density and the $\mathrm{OXT}^{+}$fiber pixel density were semi-quantitated as the total pixel density of each animal, and the data were expressed as the ratio to the density of controls. The results of all immunohistochemical analyses were saved as images and the immunoreactivities were confirmed by multiple researchers. 


\subsection{Statistical Analysis}

Statistical analyses were performed using the GraphPad Prism (version 8.4.3; GraphPad Software, San Diego, CA, USA, RRID: SCR_002798). All data are presented as the mean \pm SEM and individual dots for each animal. Statistical analyses of the optical density, and the gene expressions between sexually naïve males and sexually experienced males, were assessed using Student's unpaired $t$-test. The differences in the pERK expression ratio after sexual behavior were performed using a two-way ANOVA. When significant main effects were detected using ANOVA, the post-hoc Tukey's test was performed. Several analyses in this study were conducted in a "blinded" manner.

\section{Conclusions}

In this study, we revealed the effects of male sexual experience on $d X L$ neurons at the spinal cord level. We found that, in sexually experienced rats, approximately $50 \%$ of $d X L$ neurons were activated by both intromission (erection) and ejaculation, whereas, in naïve rats, approximately $30 \%$ of $\mathrm{dXL}$ neurons were activated by intromission and more than $50 \%$ of $\mathrm{dXL}$ neurons were activated by ejaculation. We also found that sexual experience increased GRP and OXTR expression at the protein level in $\mathrm{dXL}$ neurons. To the best of our knowledge, this is the first demonstration of the effects of sexual experience on molecular expressions in the neural circuits controlling male sexual activity in the spinal cord.

Author Contributions: Conceptualization, T.O., Y.K. and H.S.; methodology, T.O. and R.K.; software, T.O.; validation, T.O. and H.S.; formal analysis, T.O.; investigation, T.O., R.U., R.K., J.N. and T.I.; resources, T.O. and H.S.; data curation, T.O.; writing —original draft preparation, T.O. and H.S.; writing—review and editing, Y.K. and T.S.; visualization, T.O. and R.U.; supervision, Y.K. and H.S.; project administration, T.O. and H.S.; funding acquisition, T.O. and H.S. All authors have read and agreed to the published version of the manuscript.

Funding: This work was supported by Grants-in-Aid for Scientific Research from the Japan Society for the Promotion of Science (JSPS) KAKENHI (to H.S.; 15K15202, 15H05724, 15KK025708, 16H06280 (ABiS); and to T.O.; 20K15837).

Institutional Review Board Statement: The study was conducted according to the guidelines of the Declaration of Helsinki, and approved by the Institutional Ethics Committees of Okayama University (protocol code OKU-2020239 and date of approval: 16 April 2020; OKU-2017189 and date of approval: 1 April 2017) and Teikyo University of Science (protocol code 16C-013 and date of approval: 28 June 2016).

Informed Consent Statement: Not applicable.

Data Availability Statement: Not applicable.

Conflicts of Interest: The authors declare no conflict of interest.

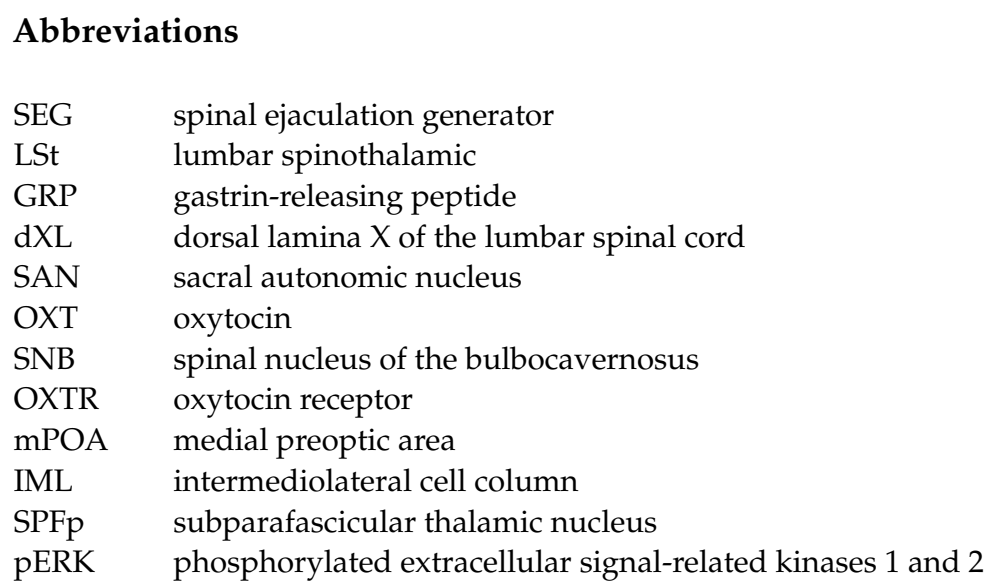




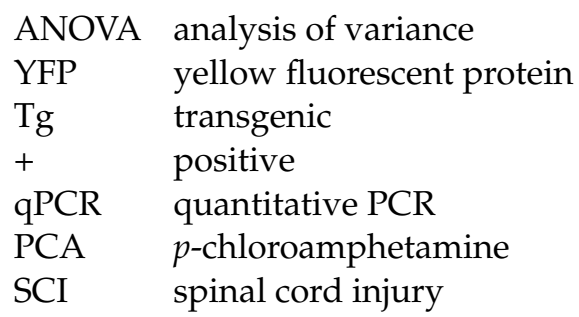

\section{References}

1. Truitt, W.A.; Coolen, L.M. Identification of a potential ejaculation generator in the spinal cord. Science 2002, 297, 1566-1569. [CrossRef]

2. Newton, B.W. Galanin-like immunoreactivity in autonomic regions of the rat lumbosacral spinal cord is sexually dimorphic and varies with the estrous cycle. Brain Res. 1992, 589, 69-83. [CrossRef]

3. Truitt, W.A.; Shipley, M.T.; Veening, J.G.; Coolen, L.M. Activation of a subset of lumbar spinothalamic neurons after copulatory behavior in male but not female rats. J. Neurosci. 2003, 23, 325-331. [CrossRef]

4. Ju, G.; Melander, T.; Ceccatelli, S.; Hokfelt, T.; Frey, P. Immunohistochemical evidence for a spinothalamic pathway co-containing cholecystokinin- and galanin-like immunoreactivities in the rat. Neuroscience 1987, 20, 439-456. [CrossRef]

5. Phan, D.C.; Newton, B.W. Cholecystokinin-8-like immunoreactivity is sexually dimorphic in a midline population of rat lumbar neurons. Neurosci. Lett. 1999, 276, 165-168. [CrossRef]

6. Nicholas, A.P.; Zhang, X.; Hokfelt, T. An immunohistochemical investigation of the opioid cell column in lamina X of the male rat lumbosacral spinal cord. Neurosci. Lett. 1999, 270, 9-12. [CrossRef]

7. Sakamoto, H.; Matsuda, K.-I.; Zuloaga, D.G.; Hongu, H.; Wada, E.; Wada, K.; Jordan, C.L.; Breedlove, S.M.; Kawata, M. Sexually dimorphic gastrin releasing peptide system in the spinal cord controls male reproductive functions. Nat. Neurosci. 2008, 11, 634-636. [CrossRef] [PubMed]

8. Sakamoto, H.; Saito, K.; Marie-Luce, C.; Raskin, K.; Oti, T.; Satoh, K.; Tamura, K.; Sakamoto, T.; Mhaouty-Kodja, S. Androgen regulates development of the sexually dimorphic gastrin-releasing peptide neuron system in the lumbar spinal cord: Evidence from a mouse line lacking androgen receptor in the nervous system. Neurosci. Lett. 2014, 558, 109-114. [CrossRef] [PubMed]

9. Oti, T.; Satoh, K.; Saito, K.; Murata, K.; Kawata, M.; Sakamoto, T.; Sakamoto, H. Three-dimensional evaluation of the spinal local neural network revealed by the high-voltage electron microscopy: A double immunohistochemical study. Histochem. Cell Biol. 2012, 138, 693-697. [CrossRef] [PubMed]

10. Tamura, K.; Kobayashi, Y.; Hirooka, A.; Takanami, K.; Oti, T.; Jogahara, T.; Oda, S.; Sakamoto, T.; Sakamoto, H. Identification of the sexually dimorphic gastrin-releasing peptide system in the lumbosacral spinal cord that controls male reproductive function in the mouse and Asian house musk shrew (Suncus murinus). J. Comp. Neurol. 2017, 525, 1586-1598. [CrossRef] [PubMed]

11. Ito, T.; Oti, T.; Takanami, K.; Satoh, K.; Ueda, Y.; Sakamoto, T.; Sakamoto, H. A sexually dimorphic peptidergic system in the lower spinal cord controlling penile function in non-human primates. Spinal Cord 2017, 56, 57-62. [CrossRef]

12. Baskerville, T.A.; Allard, J.; Wayman, C.; Douglas, A.J. Dopamine-oxytocin interactions in penile erection. Eur. J. Neurosci. 2009, 30, 2151-2164. [CrossRef] [PubMed]

13. Veronneau-Longueville, F.; Rampin, O.; Freund-Mercier, M.J.; Tang, Y.; Calas, A.; Marson, L.; McKenna, K.E.; Stoeckel, M.E.; Benoit, G.; Giuliano, F. Oxytocinergic innervation of autonomic nuclei controlling penile erection in the rat. Neuroscience 1999, 93, 1437-1447. [CrossRef]

14. Oti, T.; Satoh, K.; Uta, D.; Nagafuchi, J.; Tateishi, S.; Ueda, R.; Takanami, K.; Young, L.J.; Galione, A.; Morris, J.F.; et al. Oxytocin Influences Male Sexual Activity via Non-synaptic Axonal Release in the Spinal Cord. Curr. Biol. 2021, 31, 103-114.e5. [CrossRef] [PubMed]

15. Nutsch, V.L.; Will, R.G.; Hattori, T.; Tobiansky, D.J.; Dominguez, J.M. Sexual experience influences mating-induced activity in nitric oxide synthase-containing neurons in the medial preoptic area. Neurosci. Lett. 2014, 579, 92-96. [CrossRef] [PubMed]

16. Hosokawa, N.; Chiba, A. Effects of sexual experience on conspecific odor preference and estrous odor-induced activation of the vomeronasal projection pathway and the nucleus accumbens in male rats. Brain Res. 2005, 1066, 101-108. [CrossRef] [PubMed]

17. Giantonio, G.W.; Lund, N.L.; Gerall, A.A. Effect of diencephalic and rhinencephalic lesions on the male rat's sexual behavior. J. Comp. Physiol. Psychol. 1970, 73, 38-46. [CrossRef] [PubMed]

18. Kondo, Y. Lesions of the medial amygdala produce severe impairment of copulatory behavior in sexually inexperienced male rats. Physiol. Behav. 1992, 51, 939-943. [CrossRef]

19. Jean, A.; Bonnet, P.; Liere, P.; Mhaouty-Kodja, S.; Hardin-Pouzet, H. Revisiting medial preoptic area plasticity induced in male mice by sexual experience. Sci. Rep. 2017, 7, 17846. [CrossRef]

20. Sala, C.; Segal, M. Dendritic spines: The locus of structural and functional plasticity. Physiol. Rev. 2014, 94, 141-188. [CrossRef]

21. Gil, M.; Bhatt, R.; Picotte, K.B.; Hull, E.M. Sexual experience increases oxytocin receptor gene expression and protein in the medial preoptic area of the male rat. Psychoneuroendocrinology 2013, 38, 1688-1697. [CrossRef]

22. Yonezawa, A.; Yoshizumi, M.; Ebiko, M.; Iwanaga, T.; Kimura, Y.; Sakurada, S. Evidence for an involvement of peripheral serotonin in p-chloroamphetamine-induced ejaculation of rats. Pharmacol. Biochem. Behav. 2005, 82, 744-750. [CrossRef]

23. Sakamoto, H. Sexually dimorphic nuclei in the spinal cord control male sexual functions. Front. Neurosci. 2014, 8, 184. [CrossRef] 
24. Maejima, S.; Abe, Y.; Yamaguchi, S.; Musatov, S.; Ogawa, S.; Kondo, Y.; Tsukahara, S. VGF in the Medial Preoptic Nucleus Increases Sexual Activity Following Sexual Arousal Induction in Male Rats. Endocrinology 2018, 159, 3993-4005. [CrossRef]

25. Wiggins, J.W.; Kozyrev, N.; Sledd, J.E.; Wilson, G.G.; Coolen, L.M. Chronic Spinal Cord Injury Reduces Gastrin-Releasing Peptide in the Spinal Ejaculation Generator in Male Rats. J. Neurotrauma 2019, 36, 3378-3393. [CrossRef] [PubMed]

26. Wiggins, J.W.; Sledd, J.E.; Coolen, L.M. Spinal Cord Injury Causes Reduction of Galanin and Gastrin Releasing Peptide mRNA Expression in the Spinal Ejaculation Generator of Male Rats. Front. Neurol. 2021, 12, 982. [CrossRef]

27. Sachs, B.D.; Garinello, L.D. Hypothetical spinal pacemaker regulating penile reflexes in rats: Evidence from transection of spinal cord and dorsal penile nerves. J. Comp. Physiol. Psychol. 1980, 94, 530-535. [CrossRef]

28. Kozyrev, N.; Lehman, M.N.; Coolen, L.M. Activation of gastrin-releasing peptide receptors in the lumbosacral spinal cord is required for ejaculation in male rats. J. Sex. Med. 2012, 9, 1303-1318. [CrossRef]

29. Oti, T.; Takanami, K.; Ito, S.; Ueda, T.; Matsuda, K.I.; Kawata, M.; Soh, J.; Ukimura, O.; Sakamoto, T.; Sakamoto, H. Effects of Sex Steroids on the Spinal Gastrin-Releasing Peptide System Controlling Male Sexual Function in Rats. Endocrinology 2018, 159, 1886-1896. [CrossRef] [PubMed]

30. Oti, T.; Takanami, K.; Katayama, N.; Edey, T.; Satoh, K.; Sakamoto, T.; Sakamoto, H. Perinatal testosterone exposure is critical for the development of the male-specific sexually dimorphic gastrin-releasing peptide system in the lumbosacral spinal cord that mediates erection and ejaculation. Biol. Sex Differ. 2016, 7, 4. [CrossRef] [PubMed]

31. Takanami, K.; Sakamoto, H.; Matsuda, K.I.; Satoh, K.; Tanida, T.; Yamada, S.; Inoue, K.; Oti, T.; Sakamoto, T.; Kawata, M. Distribution of gastrin-releasing peptide in the rat trigeminal and spinal somatosensory systems. J. Comp. Neurol. 2014, 522, 1858-1873. [CrossRef]

32. Katayama, N.; Oti, T.; Takanami, K.; Sakamoto, T.; Sakamoto, H. Postnatal development of the gastrin-releasing peptide system in the lumbosacral spinal cord controlling male reproductive function in rats. Proc. Jpn. Acad. Ser. B 2016, 92, 69-75. [CrossRef]

33. Sun, Y.G.; Chen, Z.F. A gastrin-releasing peptide receptor mediates the itch sensation in the spinal cord. Nature 2007, 448, 700-703. [CrossRef]

34. Staudt, M.D.; de Oliveira, C.V.; Lehman, M.N.; McKenna, K.E.; Coolen, L.M. Activation of MAP kinase in lumbar spinothalamic cells is required for ejaculation. J. Sex. Med. 2010, 7, 2445-2457. [CrossRef] [PubMed]

35. Satoh, K.; Oti, T.; Katoh, A.; Ueta, Y.; Morris, J.F.; Sakamoto, T.; Sakamoto, H. In vivo processing and release into the circulation of GFP fusion protein in arginine vasopressin enhanced GFP transgenic rats: Response to osmotic stimulation. FEBS J. 2015, 282, 2488-2499. [CrossRef] [PubMed]

36. Castel, M.; Morris, J.F.; Whitnall, M.H.; Sivan, N. Improved visualization of the immunoreactive hypothalamo-neurohypophysial system by use of immuno-gold techniques. Cell Tissue Res. 1986, 243, 193-204. [CrossRef] [PubMed]

37. Wang, H.; Ward, A.R.; Morris, J.F. Oestradiol acutely stimulates exocytosis of oxytocin and vasopressin from dendrites and somata of hypothalamic magnocellular neurons. Neuroscience 1995, 68, 1179-1188. [CrossRef] 